\title{
Backward Variational Approach on Time Scales with an Action Depending on the Free Endpoints
}

\author{
Agnieszka B. Malinowska ${ }^{a}$ and Delfim F. M. Torres ${ }^{b}$ \\ ${ }^{a}$ Faculty of Computer Science, Białystok University of Technology, 15-351 Białystok, Poland \\ b Department of Mathematics, University of Aveiro, 3810-193 Aveiro, Portugal
}

Reprint requests to D.F.M. T.; Tel.: +351 234370668; Fax: +351 234370066; E-mail: delfim@ua.pt

Z. Naturforsch. 66a, 401 - 410 (2011); received October 19, 2010 / revised December 18, 2010

\begin{abstract}
We establish necessary optimality conditions for variational problems with an action depending on the free endpoints. New transversality conditions are also obtained. The results are formulated and proved using the recent and general theory of time scales via the backward nabla differential operator.

Key words: Calculus of Variations; Transversality Conditions; Time Scales; Backward Approach.

PACS numbers: 02.30.Xx; 02.30.Yy

Mathematics Subject Classification 2000: 49K05; 39A12
\end{abstract}

\section{Introduction}

Physics and control on an arbitrary time scale is an area of strong current research that unifies discrete, continuous, and quantum results and generalize the theory to more complex domains [1-3]. The new calculus on time scales has been applied, among others, in physics and control of population, quantum calculus, economics, communication networks, and robotic control (see [4] and references therein). The variational approach on time scales is a fertile area under strong current research [5-12]. In this paper we study problems in Lagrange form with an action functional and a velocity vector without boundary conditions $x(a)$ and $x(b)$. The considered problems are more general because of the dependence of the Hamiltonian on $x(a)$ and $x(b)$. Such possibility is not covered by the literature. Our study is done using the nabla approach to time scales, which seems promising with respect to applications (see, e.g., [13-15]). This work is motivated by the recent advancements obtained by Cruz et al. [16] and Malinowska and Torres [17] about necessary optimality conditions for the problem of the calculus of variations with a free endpoint $x(T)$ but whose Lagrangian depends explicitly on $x(T)$. Such problems seem to have important implications in physical applications [16]. In contrast to authors of [16,17], we adopt here a backward perspective, which has proved useful, and sometimes more natural and preferable, with re- spect to several applications $[13-15,18,19]$. The advantage of the here promoted backward approach becomes evident when one considers that the time scales analysis can also have important implications for numerical analysts, who often prefer backward differences rather than forward differences to handle their computations due to practical implementation reasons and also for better stability properties of implicit discretizations $[19,20]$.

The paper is organized as follows. Section 2 presents the necessary definitions and concepts of the calculus on time scales; our results are formulated, proved, and illustrated through examples in Section 3. Both Lagrangian (Section 3.1) and Hamiltonian (Section 3.2) approaches are considered. Main results of the paper include necessary optimality conditions with new transversality conditions (Theorems 3.2 and 3.9) that become sufficient under appropriate convexity assumptions (Theorem 3.14).

\section{Time Scales Calculus}

For a general introduction to the calculus on time scales we refer the reader to the books [21, 22]. Here we only give those notions and results needed in the sequel. In particular we are interested in the backward nabla differential approach to time scales [19]. As usual $\mathbb{R}, \mathbb{Z}$, and $\mathbb{N}$ denote, respectively, the set of real, integer, and natural numbers. 
A time scale $\mathbb{T}$ is an arbitrary nonempty closed subset of $\mathbb{R}$. Thus, $\mathbb{R}, \mathbb{Z}$, and $\mathbb{N}$, are trivial examples of times scales. Other examples of times scales are: $[-1,4] \cup \mathbb{N}, h \mathbb{Z}:=\{h z \mid z \in \mathbb{Z}\}$ for some $h>0$, $q^{\mathbb{N}_{0}}:=\left\{q^{k} \mid k \in \mathbb{N}_{0}\right\}$ for some $q>1$, and the Cantor set. We assume that a time scale $\mathbb{T}$ has the topology that it inherits from the real numbers with the standard topology.

The forward jump operator $\sigma: \mathbb{T} \rightarrow \mathbb{T}$ is defined by $\sigma(t)=\inf \{s \in \mathbb{T}: s>t\}$ if $t \neq \sup \mathbb{T}$, and $\sigma(\sup \mathbb{T})=$ sup $\mathbb{T}$. The backward jump operator $\rho: \mathbb{T} \rightarrow \mathbb{T}$ is defined by $\rho(t)=\sup \{s \in \mathbb{T}: s<t\}$ if $t \neq \inf \mathbb{T}$, and $\rho(\inf \mathbb{T})=\inf \mathbb{T}$.

A point $t \in \mathbb{T}$ is called right-dense, right-scattered, left-dense, and left-scattered if $\sigma(t)=t, \sigma(t)>t$, $\rho(t)=t$, and $\rho(t)<t$, respectively. We say that $t$ is isolated if $\rho(t)<t<\sigma(t)$, that $t$ is dense if $\rho(t)=$ $t=\sigma(t)$. The (backward) graininess function $v: \mathbb{T} \rightarrow$ $[0, \infty)$ is defined by $v(t)=t-\rho(t)$, for all $t \in \mathbb{T}$. Hence, for a given $t, v(t)$ measures the distance of $t$ to its left neighbour. It is clear that when $\mathbb{T}=\mathbb{R}$ one has $\sigma(t)=t=\rho(t)$, and $v(t)=0$ for any $t$. When $\mathbb{T}=\mathbb{Z}$, $\sigma(t)=t+1, \rho(t)=t-1$, and $v(t)=1$ for any $t$.

In order to introduce the definition of nabla derivative, we define a new set $\mathbb{T}_{\kappa}$ which is derived from $\mathbb{T}$ as follows: if $\mathbb{T}$ has a right-scattered minimum $m$, then $\mathbb{T}_{\kappa}=\mathbb{T} \backslash\{m\}$; otherwise, $\mathbb{T}_{\kappa}=\mathbb{T}$.

Definition 2.1. We say that a function $f: \mathbb{T} \rightarrow \mathbb{R}$ is nabla differentiable at $t \in \mathbb{T}_{\kappa}$ if there is a number $f^{\nabla}(t)$ such that for all $\varepsilon>0$ there exists a neighbourhood $U$ of $t$ (i.e., $U=] t-\delta, t+\delta[\cap \mathbb{T}$ for some $\delta>0$ ) such that

$\left|f(\rho(t))-f(s)-f^{\nabla}(t)(\rho(t)-s)\right| \leq \varepsilon|\rho(t)-s|$,

for all $s \in U$.

We call $f^{\nabla}(t)$ the nabla derivative of $f$ at $t$. Moreover, we say that $f$ is nabla differentiable on $\mathbb{T}$ provided $f^{\nabla}(t)$ exists for all $t \in \mathbb{T}_{\kappa}$.

Theorem 2.2. (Theorem 8.39 in [21]) Let $\mathbb{T}$ be a time scale, $f: \mathbb{T} \rightarrow \mathbb{R}$, and $t \in \mathbb{T}_{\kappa}$. If $f$ is nabla differentiable at $t$, then $f$ is continuous at $t$. If $f$ is continuous at $t$ and $t$ is left-scattered, then $f$ is nabla differentiable at $t$ and $f^{\nabla}(t)=\frac{f(t)-f(\rho(t))}{t-\rho(t)}$. If $t$ is left-dense, then $f$ is nabla differentiable at $t$ if and only if the limit $\lim _{s \rightarrow t} \frac{f(t)-f(s)}{t-s}$ exists as a finite number. In this case, $f^{\nabla}(t)=\lim _{s \rightarrow t} \frac{f(t)-f(s)}{t-s}$. If $f$ is nabla differentiable at $t$, then $f(\rho(t))=f(t)-v(t) f^{\nabla}(t)$.
Remark 2.3. When $\mathbb{T}=\mathbb{R}$, then $f: \mathbb{R} \rightarrow \mathbb{R}$ is nabla differentiable at $t \in \mathbb{R}$ if and only if $f^{\nabla}(t)=$ $\lim _{s \rightarrow t} \frac{f(t)-f(s)}{t-s}$ exists, i.e., if and only if $f$ is differentiable at $t$ in the ordinary sense. When $\mathbb{T}=\mathbb{Z}$, then $f: \mathbb{Z} \rightarrow \mathbb{R}$ is always nabla differentiable at $t \in \mathbb{Z}$ and $f^{\nabla}(t)=\frac{f(t)-f(\rho(t))}{t-\rho(t)}=f(t)-f(t-1)=: \nabla f(t)$, i.e., $\nabla$ is the usual backward difference operator defined by the last equation above. For any time scale $\mathbb{T}$, when $f$ is a constant, then $f^{\nabla}=0$; if $f(t)=k t$ for some constant $k$, then $f^{\nabla}=k$.

In order to simplify expressions, we denote the composition $f \circ \rho$ by $f^{\rho}$.

Theorem 2.4. (Theorem 8.41 in [21]) Suppose $f, g$ : $\mathbb{T} \rightarrow \mathbb{R}$ are nabla differentiable at $t \in \mathbb{T}_{\kappa}$. Then, the sum $f+g: \mathbb{T} \rightarrow \mathbb{R}$ is nabla differentiable at $t$ and $(f+$ $g)^{\nabla}(t)=f^{\nabla}(t)+g^{\nabla}(t) ;$ for any constant $\alpha, \alpha f: \mathbb{T} \rightarrow \mathbb{R}$ is nabla differentiable at $t$ and $(\alpha f)^{\nabla}(t)=\alpha f^{\nabla}(t)$; the product $f g: \mathbb{T} \rightarrow \mathbb{R}$ is nabla differentiable at $t$ and $(f g)^{\nabla}(t)=f^{\nabla}(t) g(t)+f^{\rho}(t) g^{\nabla}(t)=f^{\nabla}(t) g^{\rho}(t)+$ $f(t) g^{\nabla}(t)$.

Definition 2.5. Let $\mathbb{T}$ be a time scale, $f: \mathbb{T} \rightarrow \mathbb{R}$. We say that function $f$ is $v$-regressive if $1-v(t) f(t) \neq 0$ for all $t \in \mathbb{T}_{\kappa}$.

Definition 2.6. A function $F: \mathbb{T} \rightarrow \mathbb{R}$ is called a nabla antiderivative of $f: \mathbb{T} \rightarrow \mathbb{R}$ provided $F^{\nabla}(t)=f(t)$ for all $t \in \mathbb{T}_{\kappa}$. In this case we define the nabla integral of $f$ from $a$ to $b(a, b \in \mathbb{T})$ by $\int_{a}^{b} f(t) \nabla t:=F(b)-F(a)$.

In order to present a class of functions that possess a nabla antiderivative, the following definition is introduced.

Definition 2.7. Let $\mathbb{T}$ be a time scale, $f: \mathbb{T} \rightarrow \mathbb{R}$. We say that function $f$ is ld-continuous if it is continuous at left-dense points and its right-sided limits exist (finite) at all right-dense points.

Theorem 2.8. (Theorem 8.45 in [21]) Every ldcontinuous function has a nabla antiderivative. In particular, if $a \in \mathbb{T}$, then the function $F$ defined by $F(t)=$ $\int_{a}^{t} f(\tau) \nabla \tau, t \in \mathbb{T}$, is a nabla antiderivative of $f$.

The set of all ld-continuous functions $f: \mathbb{T} \rightarrow \mathbb{R}$ is denoted by $C_{\mathrm{ld}}(\mathbb{T}, \mathbb{R})$, and the set of all nabla differentiable functions with ld-continuous derivative by $C_{\mathrm{ld}}^{1}(\mathbb{T}, \mathbb{R})$. 
Theorem 2.9. (Theorem 8.46 in [21]) If $f \in C_{l d}(\mathbb{T}, \mathbb{R})$ and $t \in \mathbb{T}_{\kappa}$, then $\int_{\rho(t)}^{t} f(\tau) \nabla \tau=v(t) f(t)$.

Theorem 2.10. (Theorem 8.47 in [21]) If $a, b$, $c \in \mathbb{T}, a \leq c \leq b, \alpha \in \mathbb{R}$, and $f, g \in C_{l d}(\mathbb{T}, \mathbb{R})$, then $\int_{a}^{b}(f(t)+g(t)) \nabla t=\int_{a}^{b} f(t) \nabla t+\int_{a}^{b} g(t) \nabla t$; $\int_{a}^{b} \alpha f(t) \nabla t=\alpha \int_{a}^{b} f(t) \nabla t ; \int_{a}^{b} f(t) \nabla t=-\int_{b}^{a} f(t) \nabla t ;$ $\int_{a}^{a} f(t) \nabla t=0 ; \quad \int_{a}^{b} f(t) \nabla t=\int_{a}^{c} f(t) \nabla t+\int_{c}^{b} f(t) \nabla t$. If $f(t)>0$ for all $a<t \leq b$, then $\int_{a}^{b} f(t) \nabla t>0$; $\int_{a}^{b} f^{\rho}(t) g^{\nabla}(t) \nabla t=[(f g)(t)]_{t=a}^{t=b}-\int_{a}^{b} f^{\nabla}(t) g(t) \nabla t ;$ $\int_{a}^{b} f(t) g^{\nabla}(t) \nabla t=[(f g)(t)]_{t=a}^{t=b}-\int_{a}^{b} f^{\nabla}(t) g^{\rho}(t) \nabla t$.

Remark 2.11. Let $a, b \in \mathbb{T}$ and $f \in C_{\mathrm{ld}}(\mathbb{T}, \mathbb{R})$. For $\mathbb{T}=\mathbb{R}$, then $\int_{a}^{b} f(t) \nabla t=\int_{a}^{b} f(t) \mathrm{d} t$, where the integral on the right side is the usual Riemann integral. For $\mathbb{T}=\mathbb{Z}$, then $\int_{a}^{b} f(t) \nabla t=\sum_{t=a+1}^{b} f(t)$ if $a<b$, $\int_{a}^{b} f(t) \nabla t=0$ if $a=b$, and $\int_{a}^{b} f(t) \nabla t=-\sum_{t=b+1}^{a} f(t)$ if $a>b$.

Let $a, b \in \mathbb{T}$ with $a<b$. We define the interval $[a, b]$ in $\mathbb{T}$ by $[a, b]:=\{t \in \mathbb{T}: a \leq t \leq b\}$. Open intervals and half-open intervals in $\mathbb{T}$ are defined accordingly. Note that $[a, b]_{\kappa}=[a, b]$ if $a$ is right-dense and $[a, b]_{\kappa}=$ $[\sigma(a), b]$ if $a$ is right-scattered.

Lemma 2.12. ([18]) Let $f, g \in C_{\mathrm{ld}}([a, b], \mathbb{R})$. If $\int_{a}^{b}\left(f(t) \eta^{\rho}(t)+g(t) \eta^{\nabla}(t)\right) \nabla t=0$ for all $\eta \in$ $C_{\mathrm{ld}}^{1}([a, b], \mathbb{R})$ such that $\eta(a)=\eta(b)=0$, then $g$ is nabla differentiable and $g^{\nabla}(t)=f(t) \quad \forall t \in[a, b]_{\kappa}$.

\section{Main Results}

Throughout we let $A, B \in \mathbb{T}$ with $A<B$. Now let $[a, b]$ be a subinterval of $[A, B]$, with $a, b \in \mathbb{T}$ and $A<a$. The problem of the calculus of variations on time scales under our consideration consists of minimizing or maximizing

$$
\begin{gathered}
\mathcal{L}[x]=\int_{a}^{b} f\left(t, x^{\rho}(t), x^{\nabla}(t), x(a), x(b)\right) \nabla t, \\
\left(x(a)=x_{a}\right), \quad\left(x(b)=x_{b}\right)
\end{gathered}
$$

over all $x \in C_{\mathrm{ld}}^{1}([A, b], \mathbb{R})$. Using parentheses around the endpoint conditions means that the conditions may or may not be present. We assume that $f(t, x, v, z, s)$ : $[A, b] \times \mathbb{R}^{4} \rightarrow \mathbb{R}$ has partial continuous derivatives with respect to $x, v, z, s$ for all $t \in[A, b]$, and $f(t, \cdot, \cdot, \cdot, \cdot)$ and its partial derivatives are ld-continuous for all $t \in[A, b]$.

A function $x \in C_{\mathrm{ld}}^{1}([A, b], \mathbb{R})$ is said to be an admissible function provided that it satisfies the endpoints conditions (if any is given). Let us consider the following norm in $C_{\mathrm{ld}}^{1}([A, b], \mathbb{R}):\|x\|_{1}=\sup _{t \in[A, b]}\left|x^{\rho}(t)\right|+$ $\sup _{t \in[A, b]}\left|x^{\nabla}(t)\right|$.

Definition 3.1. An admissible function $\tilde{x}$ is said to be a weak local minimizer (respectively weak local maximizer) for (1) if there exists $\delta>0$ such that $\mathcal{L}[\tilde{x}] \leq \mathcal{L}[x]$ (respectively $\mathcal{L}[\tilde{x}] \geq \mathcal{L}[x]$ ) for all admissible $x$ with $\|x-\tilde{x}\|_{1}<\delta$.

\subsection{Lagrangian Approach}

Next theorem gives necessary optimality conditions for the problem (1).

Theorem 3.2. If $\tilde{x}$ is an extremizer (i.e., a weak local minimizer or a weak local maximizer) for the problem (1), then

$$
\begin{aligned}
& f_{x^{\nabla}}^{\nabla}\left(t, \tilde{x}^{\rho}(t), \tilde{x}^{\nabla}(t), \tilde{x}(a), \tilde{x}(b)\right) \\
& \quad=f_{x} \rho\left(t, \tilde{x}^{\rho}(t), \tilde{x}^{\nabla}(t), \tilde{x}(a), \tilde{x}(b)\right)
\end{aligned}
$$

for all $t \in[a, b]_{\kappa}$. Moreover, if $x(a)$ is not specified, then

$$
\begin{aligned}
& f_{x^{\nabla}}\left(a, \tilde{x}^{\rho}(a), \tilde{x}^{\nabla}(a), \tilde{x}(a), \tilde{x}(b)\right) \\
& \quad=\int_{a}^{b} f_{z}\left(t, \tilde{x}^{\rho}(t), \tilde{x}^{\nabla}(t), \tilde{x}(a), \tilde{x}(b)\right) \nabla t
\end{aligned}
$$

if $x(b)$ is not specified, then

$$
\begin{aligned}
& f_{x^{\nabla}}\left(b, \tilde{x}^{\rho}(b), \tilde{x}^{\nabla}(b), \tilde{x}(a), \tilde{x}(b)\right) \\
& \quad=-\int_{a}^{b} f_{s}\left(t, \tilde{x}^{\rho}(t), \tilde{x}^{\nabla}(t), \tilde{x}(a), \tilde{x}(b)\right) \nabla t .
\end{aligned}
$$

Proof. Suppose that $L$ has a weak local extremum at $\tilde{x}$. We can proceed as Lagrange did, by considering the value of $L$ at a nearby function $x=\tilde{x}+\varepsilon h$, where $\varepsilon \in \mathbb{R}$ is a small parameter, $h \in C_{\mathrm{ld}}^{1}([A, b], \mathbb{R})$. We do not require $h(a)=0$ or $h(b)=0$ in case $x(a)$ or $x(b)$, respectively, is free (it is possible that both are free). Let

$$
\begin{aligned}
\phi(\varepsilon)= & L[(\tilde{x}+\varepsilon h)(\cdot)] \\
= & \int_{a}^{b} f\left(t, \tilde{x}^{\rho}(t)+\varepsilon h(t), \tilde{x}^{\nabla}(t)\right. \\
& \left.+\varepsilon^{\nabla}(t), \tilde{x}(a)+\varepsilon h(a), \tilde{x}(b)+\varepsilon h(b)\right) \nabla t .
\end{aligned}
$$


A necessary condition for $\tilde{x}$ to be an extremizer is given by

$$
\begin{aligned}
& \left.\phi^{\prime}(\varepsilon)\right|_{\varepsilon=0}=0 \\
& \Leftrightarrow \int_{a}^{b}\left[f_{x} \rho(\cdots) h^{\rho}(t)+f_{x} \nabla(\cdots) h^{\nabla}(t)\right. \\
& \left.\quad+f_{z}(\cdots) h(a)+f_{s}(\cdots) h(b)\right] \Delta t=0,
\end{aligned}
$$

where $(\cdots)=\left(t, \tilde{x}^{\rho}(t), \tilde{x}^{\nabla}(t), \tilde{x}(a), \tilde{x}(b)\right)$. Integration by parts gives

$$
\begin{aligned}
0= & \int_{a}^{b}\left(f_{x} \rho(\cdots)-f_{x^{\nabla}}^{\nabla}(\cdots)\right) h^{\rho}(t) \nabla t \\
& +h(b)\left(\left.f_{x^{\nabla}}(\cdots)\right|_{t=b}+\int_{a}^{b} f_{s}(\cdots) \nabla t\right) \\
& +h(a)\left(-\left.f_{x}(\cdots)\right|_{t=a}+\int_{a}^{b} f_{z}(\cdots) \nabla t\right) .
\end{aligned}
$$

We first consider functions $h(t)$ such that $h(a)=$ $h(b)=0$. Then, by Lemma 2.12 , we have

$$
f_{x} \rho(\cdots)-f_{x^{\nabla}}^{\nabla}(\cdots)=0
$$

for all $t \in[a, b]_{\kappa}$. Therefore, in order for $\tilde{x}$ to be an extremizer for the problem (1), $\tilde{x}$ must be a solution of the nabla differential Euler-Lagrange equation. But if $\tilde{x}$ is a solution of (7), the first integral in expression (6) vanishes, and then the condition (5) takes the form

$$
\begin{aligned}
& h(b)\left(\left.f_{x} \nabla(\cdots)\right|_{t=b}+\int_{a}^{b} f_{s}(\cdots) \nabla t\right) \\
& +h(a)\left(-\left.f_{x} \nabla(\cdots)\right|_{t=a}+\int_{a}^{b} f_{z}(\cdots) \nabla t\right)=0 .
\end{aligned}
$$

If $x(a)=x_{a}$ and $x(b)=x_{b}$ are given in the formulation of problem (1), then the latter equation is trivially satisfied since $h(a)=h(b)=0$. When $x(a)$ is free, then (3) holds; when $x(b)$ is free, then (4) holds; since $h(a)$ or $h(b)$ is, respectively, arbitrary.

Letting $\mathbb{T}=\mathbb{R}$ in Theorem 3.2 we immediately obtain the corresponding result in the classical context of the calculus of variations.

Corollary 3.3. (cf. $[16,17]$ ) If $\tilde{x}$ is an extremizer for

$$
\begin{gathered}
\mathcal{L}[x]=\int_{a}^{b} f\left(t, x(t), x^{\prime}(t), x(a), x(b)\right) \mathrm{d} t \\
\left(x(a)=x_{a}\right), \quad\left(x(b)=x_{b}\right)
\end{gathered}
$$

then

$$
\begin{aligned}
& \frac{\mathrm{d}}{\mathrm{d} t} f_{x^{\prime}}\left(t, \tilde{x}(t), \tilde{x}^{\prime}(t), \tilde{x}(a), \tilde{x}(b)\right) \\
& \quad=f_{x}\left(t, \tilde{x}(t), \tilde{x}^{\prime}(t), \tilde{x}(a), \tilde{x}(b)\right)
\end{aligned}
$$

for all $t \in[a, b]$. Moreover, if $x(a)$ is free, then

$$
\begin{aligned}
f_{x^{\prime}} & \left(a, \tilde{x}(a), \tilde{x}^{\prime}(a), \tilde{x}(a), \tilde{x}(b)\right) \\
& =\int_{a}^{b} f_{z}\left(t, \tilde{x}(t), \tilde{x}^{\prime}(t), \tilde{x}(a), \tilde{x}(b)\right) \mathrm{d} t
\end{aligned}
$$

if $x(b)$ is free, then

$$
\begin{aligned}
& f_{x^{\prime}}\left(b, \tilde{x}(b), \tilde{x}^{\prime}(b), \tilde{x}(a), \tilde{x}(b)\right) \\
& \quad=-\int_{a}^{b} f_{s}\left(t, \tilde{x}(t), \tilde{x}^{\prime}(t), \tilde{x}(a), \tilde{x}(b)\right) \mathrm{d} t
\end{aligned}
$$

Example 3.4. Consider a river with parallel straight banks, $b$ units apart. One of the banks coincides with the $y$-axis, the water is assumed to be moving parallel to the banks with speed $v$ that depends, as usual, on the $x$-coordinate, but also on the arrival point $y(b)(y(b)$ is not given and is part of the solution of the problem). A boat with constant speed $c\left(c^{2}>v^{2}\right)$ in still water is crossing the river in the shortest possible time, using the point $y(0)=0$ as point of departure. The endpoint $y(b)$ is allowed to move freely along the other bank $x=b$. Then one can easily obtain that the time of passage along the path $y(x)$ is given by

$$
\begin{aligned}
\mathcal{T}[y]= & \int_{0}^{b}\left(\sqrt{c^{2}\left(1+\left(y^{\prime}(x)\right)^{2}\right)-v^{2}(x, y(b))}\right. \\
& \left.-v(x, y(b)) y^{\prime}(x)\right)\left(c^{2}-v^{2}(x, y(b))\right)^{-1} \mathrm{~d} x,
\end{aligned}
$$

where $v=v(x, y(b))$ is a known function of $x$ and $y(b)$. This is not a standard problem because the integrand depends on $y(b)$. Corollary 3.3 gives the solution.

Remark 3.5. In the classical setting $f$ does not depend on $x(a)$ and $x(b)$, i.e., $f_{z}=0$ and $f_{s}=0$. In that case (8) and (9) reduce to the well known natural boundary conditions $f_{x^{\prime}}\left(a, \tilde{x}(a), \tilde{x}^{\prime}(a)\right)=0$ and $f_{x^{\prime}}\left(b, \tilde{x}(b), \tilde{x}^{\prime}(b)\right)$ $=0$.

Similarly, we can obtain other corollaries by choosing different time scales. The next corollary is obtained from Theorem 3.2 letting $\mathbb{T}=\mathbb{Z}$. 
Corollary 3.6. If $\tilde{x}$ is an extremizer for

$$
\begin{gathered}
L[x]=\sum_{t=a+1}^{b} f(t, x(t-1), \nabla x(t), x(a), x(b)), \\
\left(x(a)=x_{a}\right), \quad\left(x(b)=x_{b}\right),
\end{gathered}
$$

then $f_{x}(t, \tilde{x}(t-1), \nabla \tilde{x}(t), \tilde{x}(a), \tilde{x}(b))=\nabla f_{v}(t, \tilde{x}(t-1)$, $\nabla \tilde{x}(t), \tilde{x}(a), \tilde{x}(b))$ for all $t \in[a+1, b]$. Moreover,

$$
\begin{aligned}
f_{v}(a, \tilde{x}(a-1), \nabla \tilde{x}(a), \tilde{x}(a), \tilde{x}(b)) \\
\quad=\sum_{t=a+1}^{b} f_{z}(t, \tilde{x}(t-1), \nabla \tilde{x}(t), \tilde{x}(a), \tilde{x}(b)),
\end{aligned}
$$

if $x(a)$ is not specified and

$$
\begin{aligned}
& f_{v}(b, \tilde{x}(b-1), \nabla \tilde{x}(b), \tilde{x}(a), \tilde{x}(b)) \\
& \quad=-\sum_{t=a+1}^{b} f_{s}(t, \tilde{x}(t-1), \nabla \tilde{x}(t), \tilde{x}(a), \tilde{x}(b)),
\end{aligned}
$$

if $x(b)$ is not specified.

Let $\mathbb{T}=q^{\mathbb{N}_{0}}, q>1$. To simplify notation, we use $\nabla_{q}$ for the $q$-nabla derivative $\nabla_{q} x(t)=\frac{x(t)-x\left(t q^{-1}\right)}{t\left(1-q^{-1}\right)}$.

Corollary 3.7. If $\tilde{x}$ is an extremizer for

$L[x]=\left(1-q^{-1}\right) \sum_{t \in(a, b]} t f\left(t, x\left(q^{-1} t\right), \nabla_{q} x(t), x(a), x(b)\right)$, $\left(x(a)=x_{a}\right),\left(x(b)=x_{b}\right)$,

then $f_{x}\left(t, \tilde{x}\left(q^{-1} t\right), \nabla_{q} \tilde{x}(t), \tilde{x}(a), \tilde{x}(b)\right)=\nabla_{q} f_{v}\left(t, \tilde{x}\left(q^{-1} t\right)\right.$, $\left.\nabla_{q} \tilde{x}(t), \tilde{x}(a), \tilde{x}(b)\right)$ for all $t \in(a, b]$. Moreover, if $x(a)$ is free, then

$$
\begin{aligned}
& f_{v}\left(a, \tilde{x}\left(a q^{-1}\right), \nabla_{q} \tilde{x}(a), \tilde{x}(a), \tilde{x}(b)\right) \\
& \quad=\left(1-q^{-1}\right) \sum_{t \in(a, b]} t f_{z}\left(t, \tilde{x}\left(q^{-1} t\right), \nabla_{q} \tilde{x}(t), \tilde{x}(a), \tilde{x}(b)\right)
\end{aligned}
$$

if $x(b)$ is free, then

$$
\begin{aligned}
& f_{v}\left(b, \tilde{x}\left(b q^{-1}\right), \nabla_{q} \tilde{x}(b), \tilde{x}(a), \tilde{x}(b)\right) \\
& =-\left(1-q^{-1}\right) \sum_{t \in(a, b]} t f_{s}\left(t, \tilde{x}\left(q^{-1} t\right), \nabla_{q} \tilde{x}(t), \tilde{x}(a), \tilde{x}(b)\right) .
\end{aligned}
$$

We illustrate the application of Theorem 3.2 with an example.

Example 3.8. Consider the problem

minimize $\mathcal{L}[x]=\int_{0}^{1}\left(\left(x^{\nabla}(t)\right)^{2}\right.$

$$
\left.+\alpha x^{2}(0)+\beta(x(1)-1)^{2}\right) \nabla t
$$

where $\alpha, \beta \in \mathbb{R}^{+}$. If $\tilde{x}$ is a local minimizer of (10), then conditions (2) $-(4)$ must hold, i.e.,

$$
\begin{aligned}
& \left(2 \tilde{x}^{\nabla}(t)\right)^{\nabla}=0, \\
& 2 \tilde{x}^{\nabla}(0)=\int_{0}^{1} 2 \alpha x(0) \nabla t, \\
& 2 \tilde{x}^{\nabla}(1)=-\int_{0}^{1} 2 \beta(x(1)-1) \nabla t .
\end{aligned}
$$

Equation (11) implies that there exists a constant $c \in \mathbb{R}$ such that $\tilde{x}^{\nabla}(t)=c$. Solving this equation we obtain $\tilde{x}(t)=c t+\tilde{x}(0)$. In order to determine $c$ and $\tilde{x}(0)$ we use the natural boundary conditions (12) which we can now rewrite as a system of two equations:

$$
c-\alpha \tilde{x}(0)=0, c+\beta(c+\tilde{x}(0)-1)=0 .
$$

The solution of (13) is $c=\frac{\alpha \beta}{\alpha+\beta+\alpha \beta}$ and $\tilde{x}(0)=$ $\frac{\beta}{\alpha+\beta+\alpha \beta}$. Hence, $\tilde{x}(t)=c(\alpha, \beta) t+\tilde{x}(0, \alpha, \beta)$ is a candidate for minimizer (see Fig. 1). We note that $\lim _{\alpha, \beta \rightarrow \infty} c(\alpha, \beta)=1, \lim _{\alpha, \beta \rightarrow \infty} \tilde{x}(0, \alpha, \beta)=0$, and in the limit $\alpha, \beta \rightarrow \infty$ the solution of (10) coincides with the solution of the following problem with fixed initial and terminal points: $\min \mathcal{L}[x]=\int_{0}^{1}\left(x^{\nabla}(t)\right)^{2} \nabla t$, subject to $x(0)=0$ and $x(1)=1$. Expression $\alpha x^{2}(0)+$

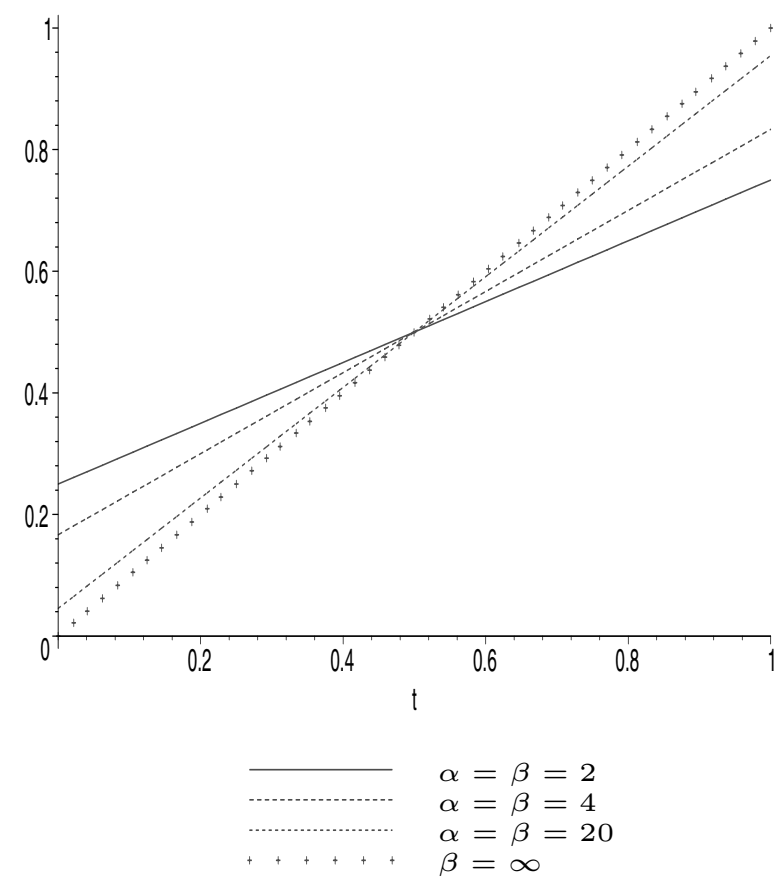

Fig. 1. Extremal $\tilde{x}(t)=c(\alpha, \beta) t+\tilde{x}(0, \alpha, \beta)$ of Example 3.8 for different values of parameters $\alpha$ and $\beta$. 
$\beta(x(1)-1)^{2}$ added to the Lagrangian $\left(x^{\nabla}(t)\right)^{2}$ works like a penalty function when $\alpha$ and $\beta$ go to infinity. The penalty function itself grows and forces the merit function (10) to increase in value when the constraints $x(0)=0$ and $x(1)=1$ are violated, and causes no growth when constraints are fulfilled.

\subsection{Hamiltonian Approach}

Now let us consider the more general variational problem of optimal control on time scales: to minimize (maximize) the functional

$\mathcal{L}[x, u]=\int_{a}^{b} f\left(t, x^{\rho}(t), u^{\rho}(t), x(a), x(b)\right) \nabla t$,

subject to

$$
\begin{array}{r}
x^{\nabla}(t)=g\left(t, x^{\rho}(t), u^{\rho}(t), x(a), x(b)\right), \\
\left(x(a)=x_{a}\right), \quad\left(x(b)=x_{b}\right),
\end{array}
$$

where $x_{a}, x_{b} \in \mathbb{R}, f(t, x, v, z, s):[A, b] \times \mathbb{R}^{4} \rightarrow \mathbb{R}$ and $g(t, x, v, z, s):[A, b] \times \mathbb{R}^{4} \rightarrow \mathbb{R}$ have partial continuous derivatives with respect to $x, v, z, s$ for all $t \in[A, b]$, and $f(t, \cdot, \cdot, \cdot, \cdot), g(t, \cdot, \cdot, \cdot, \cdot)$ and their partial derivatives are ld-continuous for all $t$. We also assume that the function $g_{x}$ is $v$-regressive.

A necessary optimality condition for problem (14) - (15) can be obtained from a general Lagrange multiplier theorem in space of infinite dimension. We form a Lagrange function $f+\lambda^{\rho}\left(g-x^{\nabla}\right)$ by introducing a multiplier $\lambda:[A, b] \rightarrow \mathbb{R}$. In what follows we shall assume that $\lambda^{\rho}$ is a nabla differentiable function on $[a, b]$. For examples of time scales for which the composition of a nabla differentiable function with $\rho$ is not nabla differentiable, we refer the reader to [21]. Note that we are interested in the study of normal extremizers only. In general one needs to replace $f$ in $f+\lambda^{\rho}\left(g-x^{\nabla}\right)$ by $\lambda_{0} f$. Normal extremizers correspond to $\lambda_{0}=1$ while abnormal ones correspond to $\lambda_{0}=0$.

Theorem 3.9. If $(\tilde{x}, \tilde{u})$ is a normal extremizer for the problem (14)-(15), then there exists a function $\tilde{p}$ such that the triple $(\tilde{x}, \tilde{u}, \tilde{p})$ satisfies the Hamiltonian system

$x^{\nabla}(t)=H_{p}\left(t, x^{\rho}(t), u^{\rho}(t), p(t), x(a), x(b)\right)$,

$(p(t))^{\nabla}=-H_{x} \rho\left(t, x^{\rho}(t), u^{\rho}(t), p(t), x(a), x(b)\right)$,

the stationary condition

$H_{u} \rho\left(t, x^{\rho}(t), u^{\rho}(t), p(t), x(a), x(b)\right)=0$, for all $t \in[a, b]_{\kappa}$, and the transversality condition

$p(a)=-\int_{a}^{b} H_{z}\left(t, x^{\rho}(t), u^{\rho}(t), p(t), x(a), x(b)\right) \nabla t$,

when $x(a)$ is free; the transversality condition

$p(b)=\int_{a}^{b} H_{s}\left(t, x^{\rho}(t), u^{\rho}(t), p(t), x(a), x(b)\right) \nabla t$,

when $x(b)$ is free, where the Hamiltonian $H(t, x, v, p, z, s):[A, b] \times \mathbb{R}^{5} \rightarrow \mathbb{R}$ is defined by

$$
\begin{aligned}
H\left(t, x^{\rho}, u^{\rho}, p, x(a), x(b)\right)= & f\left(t, x^{\rho}, u^{\rho}, x(a), x(b)\right) \\
& +p g\left(t, x^{\rho}, u^{\rho}, x(a), x(b)\right) .
\end{aligned}
$$

Proof. Let $(\tilde{x}, \tilde{u})$ be a normal extremizer for the problem (14) - (15). Using the Lagrange multiplier rule, we form the expression $\lambda^{\rho}\left(g-x^{\nabla}\right)$ for each value of $t$ (we are assuming that $\mathbb{T}$ is a time scale for which $\lambda^{\rho}$ is a nabla differentiable function on $[a, b]$ ). The replacement of $f$ by $f+\lambda^{\rho}\left(g-x^{\nabla}\right)$ in the objective functional gives us a new problem: minimize (maximize)

$$
\begin{aligned}
\mathcal{I}[x, u, \lambda]=\int_{a}^{b} & \left\{f\left(t, x^{\rho}(t), u^{\rho}(t), x(a), x(b)\right)\right. \\
& +\lambda^{\rho}(t)\left[g\left(t, x^{\rho}(t), u^{\rho}(t), x(a), x(b)\right)\right. \\
& \left.\left.-x^{\nabla}(t)\right]\right\} \nabla t, \\
& \left(x(a)=x_{a}\right), \quad\left(x(b)=x_{b}\right) .
\end{aligned}
$$

Substituting

$$
\begin{aligned}
& H\left(t, x^{\rho}, u^{\rho}, \lambda^{\rho}, x(a), x(b)\right) \\
& \quad=f\left(t, x^{\rho}, u^{\rho}, x(a), x(b)\right)+\lambda^{\rho} g\left(t, x^{\rho}, u^{\rho}, x(a), x(b)\right)
\end{aligned}
$$

into (21), we can simplify the new functional to the form

$$
\begin{gathered}
\mathcal{I}[x, u, \lambda]=\int_{a}^{b}\left[H\left(t, x^{\rho}, u^{\rho}, \lambda^{\rho}, x(a), x(b)\right)\right. \\
\left.-\lambda^{\rho}(t) x^{\nabla}(t)\right] \nabla t .
\end{gathered}
$$

The choice of $\lambda^{\rho}$ will produce no effect on the value of the functional $\mathcal{I}$, as long as the equation $x^{\nabla}(t)=$ $g\left(t, x^{\rho}(t), u^{\rho}(t), x(a), x(b)\right)$ is satisfied, i.e., as long as

$x^{\nabla}(t)=H_{\lambda \rho}\left(t, x^{\rho}(t), u^{\rho}(t), \lambda^{\rho}(t), x(a), x(b)\right)$.

Therefore, we impose (23) as a necessary condition for the minimizing (maximizing) of the functional $\mathcal{I}$. Un- 
der condition (23) the free extremum of the $\mathcal{I}$ is identical with the constrained extremum of the functional $\mathcal{L}$. In view of (22), applying Theorem 3.2 to the problem (21) gives

$$
\begin{aligned}
& \left(\lambda^{\rho}(t)\right)^{\nabla}=-H_{x} \rho\left(t, x^{\rho}(t), u^{\rho}(t), \lambda^{\rho}(t), x(a), x(b)\right), \\
& H_{u^{\rho}}\left(t, x^{\rho}(t), u^{\rho}(t), \lambda^{\rho}(t), x(a), x(b)\right)=0
\end{aligned}
$$

for all $t \in[a, b]_{\kappa}$, and the transversality conditions

$\lambda^{\rho}(a)=-\int_{a}^{b} H_{z}\left(t, x^{\rho}(t), u^{\rho}(t), \lambda^{\rho}(t), x(a), x(b)\right) \nabla t$,

$\lambda^{\rho}(b)=\int_{a}^{b} H_{s}\left(t, x^{\rho}(t), u^{\rho}(t), \lambda^{\rho}(t), x(a), x(b)\right) \nabla t$,

in case $x(a)$ and $x(b)$ are free. Note that (24) is a first order nonhomogeneous linear equation and from the assumptions on $f$ and $g$, the solution $\tilde{\lambda}^{\rho}$ exists (see Theorem 3.42 in [22]). Therefore the triple $\left(\tilde{x}, \tilde{u}, \tilde{\lambda}^{\rho}\right)$ satisfies the system $(23)-(25)$ and the transversality conditions (26) in case $x(a)$ and $x(b)$ are free. Putting $\tilde{p}=\tilde{\lambda}^{\rho}$ we obtain the intended conditions (16) - (20).

Remark 3.10. Theorem 3.9 covers the case when $(\tilde{x}, \tilde{u})$ is a normal extremizer for the problem $(14)-(15)$. We do not consider problems with abnormal extremizers, but in general such extremizers are possible. Let us consider the problem

$$
\begin{gathered}
\operatorname{minimize} \mathcal{L}[x, u]=\int_{0}^{1}(u(t))^{2} \mathrm{~d} t, \\
x^{\prime}(t)=0, \\
x(0)=0, x(1)=0
\end{gathered}
$$

defined on $\mathbb{T}=\mathbb{R}$. Then, the pair $(\tilde{x}(t), \tilde{u}(t))=(0,0)$ is abnormal minimizer for this problem. Observe that $\mathcal{I}[\tilde{x}(t), \tilde{u}(t), \lambda]=0$ for all $\lambda \in C^{1}([0,1], \mathbb{R})$. However, for the triple $(x(t), u(t), \lambda(t))=\left(t^{2}-t, 0,2 t-1\right)$ we have $\mathcal{I}[x(t), u(t), \lambda(t)]=\int_{0}^{1}-(2 t-1)^{2} \mathrm{~d} t=-\frac{1}{3}<0$.

Example 3.11. Consider the problem

$$
\begin{gathered}
\operatorname{minimize} \mathcal{L}[x, u]=\int_{0}^{3}\left(u^{\rho}(t)\right)^{2}+t^{2}(x(3)-1)^{2} \\
+t^{2}(x(0)-1)^{2} \nabla t, \\
x^{\nabla}(t)=u^{\rho}(t) .
\end{gathered}
$$

To find candidate solutions for the problem, we start by forming the Hamiltonian function

$$
\begin{aligned}
& H\left(t, x^{\rho}, u^{\rho}, p, x(0), x(3)\right) \\
& \quad=\left(u^{\rho}\right)^{2}+t^{2}(x(3)-1)^{2}+t^{2}(x(0)-1)^{2}+p u^{\rho} .
\end{aligned}
$$

Candidate solutions $(\tilde{x}, \tilde{u})$ are those satisfying the following conditions:

$$
\begin{aligned}
& (p(t))^{\nabla}=0, u^{\rho}(t)=x^{\nabla}(t), \\
& 2 u^{\rho}(t)+p(t)=0, \\
& p(0)=-\int_{0}^{3} 2 t^{2}(x(0)-1) \nabla t, \\
& p(3)=\int_{0}^{3} 2 t^{2}(x(3)-1) \nabla t .
\end{aligned}
$$

From (29) we conclude that $p(t)=c$ and a possible solution is $\tilde{x}(t)=-\frac{c}{2} t+d$, where $c, d$ are constants of nabla integration. In order to determine $c$ and $d$, we use the transversality conditions (30) that we can write as

$$
\begin{aligned}
& c=-\int_{0}^{3} 2 t^{2}(d-1) \nabla t \\
& c=\int_{0}^{3} 2 t^{2}\left(-\frac{3 c}{2}+d-1\right) \nabla t .
\end{aligned}
$$

The values of the nabla integrals in (31) depend on the time scale. Notwithstanding this fact, substituting $\int_{0}^{3} t^{2} \nabla t=k, k \in \mathbb{R}$, into (31) we can simplify the equations to the form

$$
c=-2 k(d-1), c=2 k\left(-\frac{3 c}{2}+d-1\right) \text {. }
$$

Equations (32) yield $c=0$ and $d=1$. Therefore, the extremal of the problem (28) is $\tilde{x}(t)=1$ on any time scale.

When $\mathbb{T}=\mathbb{R}$ we obtain from Theorem 3.9 the following corollary.

Corollary 3.12. Let $(\tilde{x}, \tilde{u})$ be a normal extremizer for

$$
L[x, u]=\int_{a}^{b} f(t, x(t), u(t), x(a), x(b)) \mathrm{d} t
$$

subject to

$$
\begin{gathered}
x^{\prime}(t)=g(t, x(t), u(t), x(a), x(b)) \\
\left(x(a)=x_{a}\right)\left(x(b)=x_{b}\right),
\end{gathered}
$$


where $a, b \in \mathbb{R}, a<b$. Then there exists a function $\tilde{p}$ such that the triple $(\tilde{x}, \tilde{u}, \tilde{p})$ satisfies the Hamiltonian system

$$
x^{\prime}(t)=H_{\mathcal{L}}, p^{\prime}(t)=-H_{x},
$$

the stationary condition

$$
H_{u}=0,
$$

for all $t \in[a, b]$ and the transversality condition

$$
p(a)=-\int_{a}^{b} H_{z} \mathrm{~d} t
$$

when $x(a)$ is free; the transversality condition

$$
p(b)=\int_{a}^{b} H_{s} \mathrm{~d} t,
$$

when $x(b)$ is free, where the Hamiltonian $H$ is defined by

$$
H(t, x, u, p, z, s)=f(t, x, u, z, s)+p g(t, x, u, z, s) .
$$

We illustrate the use of Corollary 3.12 with an example.

Example 3.13. Consider the problem

$$
\begin{gathered}
\operatorname{minimize} \mathcal{L}[x, u]=\int_{-1}^{1}(u(t))^{2} \mathrm{~d} t \\
x^{\prime}(t)=u(t)+x(-1) t+x(1) t
\end{gathered}
$$

We begin by writing the Hamiltonian function

$H(t, x, u, p, x(-1), x(1))=u^{2}+p(u+x(-1) t+x(1) t)$.

Candidate solutions $(\tilde{x}, \tilde{u})$ are those satisfying the following conditions:

$$
\begin{aligned}
& p^{\prime}(t)=0, \\
& x^{\prime}(t)=u(t)+x(-1) t+x(1) t, \\
& 2 u(t)+p(t)=0, \\
& p(-1)=-\int_{-1}^{1} p(t) t \mathrm{~d} t, \\
& p(1)=\int_{-1}^{1} p(t) t \mathrm{~d} t .
\end{aligned}
$$

Equation (34) has the solution $\tilde{p}(t)=c,-1 \leq t \leq 1$, which upon substitution into (37) yields

$$
c=\int_{-1}^{1} c t \mathrm{~d} t=0 .
$$

From the stationary condition (36) we get $\tilde{u}(t)=0$. Therefore, $\mathcal{L}[\tilde{x}, \tilde{u}]=0$. Finally, substituting the optimal control candidate back into (35) yields

$$
\tilde{x}^{\prime}(t)=\tilde{x}(-1) t+\tilde{x}(1) t
$$

Integrating (38), we obtain

$$
\tilde{x}(t)=\frac{1}{2} t^{2}(\tilde{x}(-1)+\tilde{x}(1))+d .
$$

Substituting $t=1$ and $t=-1$ into (39), we get $d=0$ and $\tilde{x}(-1)=\tilde{x}(1)$. Therefore, extremals of the problem (33) are $\tilde{x}(t)=t^{2} \tilde{x}(1)$, where $\tilde{x}(1)$ is any real number.

Theorem 3.14. Let $\left(x^{\rho}, u^{\rho}, z, s\right) \rightarrow f\left(t, x^{\rho}, u^{\rho}, z, s\right)$ and $\left(x^{\rho}, u^{\rho}, z, s\right) \rightarrow g\left(t, x^{\rho}, u^{\rho}, z, s\right)$ be jointly convex (concave) in $\left(x^{\rho}, u^{\rho}, z, s\right)$ for any $t$. If $(\tilde{x}, \tilde{u}, \tilde{p})$ is a solution of system (16) $-(20)$ and $\tilde{p}(t) \geq 0$ for all $t \in[a, b]$, then $(\tilde{x}, \tilde{u})$ is a global minimizer (maximizer) of problem (14)-(15).

Proof. We shall give the proof for the convex case. Since $f$ is jointly convex in $\left(x^{\rho}, u^{\rho}, z, s\right)$ for any admissible pair $(x, u)$, we have

$$
\begin{aligned}
& \mathcal{L}[x, u]-\mathcal{L}[\tilde{x}, \tilde{u}] \\
& \begin{aligned}
\int_{a}^{b}[ & f\left(t, x^{\rho}(t), u^{\rho}(t), x(a), x(b)\right) \\
& \left.\quad-f\left(t, \tilde{x}^{\rho}(t), \tilde{u}^{\rho}(t), \tilde{x}(a), \tilde{x}(b)\right)\right] \nabla t \\
\geq \int_{a}^{b}[ & f_{x} \rho\left(t, \tilde{x}^{\rho}(t), \tilde{u}^{\rho}(t), \tilde{x}(a), \tilde{x}(b)\right)\left(x^{\rho}(t)-\tilde{x}^{\rho}(t)\right) \\
& +f_{u^{\rho}}\left(t, \tilde{x}^{\rho}(t), \tilde{u}^{\rho}(t), \tilde{x}(a), \tilde{x}(b)\right)\left(u^{\rho}(t)-\tilde{u}^{\rho}(t)\right) \\
& +f_{z}\left(t, \tilde{x}^{\rho}(t), \tilde{u}^{\rho}(t), \tilde{x}(a), \tilde{x}(b)\right)(x(a)-\tilde{x}(a)) \\
& \left.+f_{s}\left(t, \tilde{x}^{\rho}(t), \tilde{u}^{\rho}(t), \tilde{x}(a), \tilde{x}(b)\right)(x(b)-\tilde{x}(b))\right] \nabla t .
\end{aligned}
\end{aligned}
$$

Because the triple $(\tilde{x}, \tilde{u}, \tilde{p})$ satisfies $(17)-(20)$, we obtain

$$
\begin{aligned}
\mathcal{L}[x, u] & -\mathcal{L}[\tilde{x}, \tilde{u}] \\
\geq \int_{a}^{b}[ & -\tilde{p}(t) g_{x}(\cdots)\left(x^{\rho}(t)-\tilde{x}^{\rho}(t)\right) \\
& -(\tilde{p}(t))^{\Delta}\left(x^{\rho}(t)-\tilde{x}^{\rho}(t)\right) \\
& -\tilde{p}(t) g_{u^{\rho}}(\cdots)\left(u^{\rho}(t)-\tilde{u}^{\rho}(t)\right) \\
& -\tilde{p}(t) g_{z}(\cdots)(x(a)-\tilde{x}(a)) \\
& \left.-\tilde{p}(t) g_{s}(\cdots)(x(b)-\tilde{x}(b))\right] \nabla t \\
& +\tilde{p}(b)(x(b)-\tilde{x}(b))-\tilde{p}(a)(x(a)-\tilde{x}(a)),
\end{aligned}
$$


where $(\cdots)=\left(t, \tilde{x}^{\rho}(t), \tilde{u}^{\rho}(t), \tilde{x}(a), \tilde{x}(b)\right)$. Integrating by parts the term in $(\tilde{p})^{\Delta}$, we get

$$
\begin{aligned}
& \mathcal{L}[x, u]-\mathcal{L}[\tilde{x}, \tilde{u}] \\
& \geq \int_{a}^{b} \tilde{p}(t)\left[x^{\nabla}(t)-\tilde{x}^{\nabla}(t)-g_{x} \rho(\cdots)\left(x^{\rho}(t)-\tilde{x}^{\rho}(t)\right)\right. \\
& -g_{u^{\rho}}(\cdots)\left(u^{\rho}(t)-\tilde{u}^{\rho}(t)\right)-g_{z}(\cdots)(x(a)-\tilde{x}(a)) \\
& \left.-g_{s}(\cdots)(x(b)-\tilde{x}(b))\right] \nabla t \text {. }
\end{aligned}
$$

Using (16), we obtain

$$
\begin{aligned}
\mathcal{L}[x, u] & -\mathcal{L}[\tilde{x}, \tilde{u}] \\
\geq \int_{a}^{b} \tilde{p}(t)\left[g\left(t, x^{\rho}(t), u^{\rho}(t), x(a), x(b)\right)\right. & \\
& -g\left(t, \tilde{x}^{\rho}(t), \tilde{u}^{\rho}(t), \tilde{x}(a), \tilde{x}(b)\right) \\
& -g_{x}(\cdots)\left(x^{\rho}(t)-\tilde{x}^{\rho}(t)\right) \\
& -g_{u^{\rho}}(\cdots)\left(u^{\rho}(t)-\tilde{u}^{\rho}(t)\right)-g_{z}(\cdots)(x(a)-\tilde{x}(a)) \\
& \left.-g_{s}(\cdots)(x(b)-\tilde{x}(b))\right] \nabla t .
\end{aligned}
$$

Note that the integrand is positive due to $\tilde{p}(t) \geq 0$ for all $t \in[a, b]$ and joint convexity of $g$ in $\left(x^{\sigma}, u^{\sigma}, z, s\right)$. We conclude that $\mathcal{L}[x, u] \geq \mathcal{L}[\tilde{x}, \tilde{u}]$ for each admissible pair $(x, u)$.

Example 3.15. Consider the problem (33) in Example 3.13. The integrand is independent of $(x, z, s)$ and convex in $u$. The right-hand side of the control system

[1] R. Almeida and D. F. M. Torres, Lett. Math. Phys. 92, 221-229 (2010).

[2] Z. Bartosiewicz and E. Pawluszewicz, IEEE Trans. Autom. Control 53, 571-575 (2008).

[3] E. Pawłuszewicz and D. F. M. Torres, J. Optim. Theory Appl. 145, 527-542 (2010).

[4] J. Seiffertt, S. Sanyal, and D. C. Wunsch, IEEE Trans. Syst. Man Cybern., Part B: Cybern. 38, 918-923 (2008).

[5] Z. Bartosiewicz, N. Martins, and D. F. M. Torres, Eur. J. Control 17, 9-18 (2011).

[6] M. J. Bohner, R. A. C. Ferreira, and D. F. M. Torres, Math. Inequal. Appl. 13, 511-522 (2010).

[7] R. A. C. Ferreira and D. F. M. Torres, Int. J. Ecol. Econ. Stat. 9, 65-73 (2007)

[8] R. A. C. Ferreira and D. F. M. Torres, Higher-order calculus of variations on time scales, in Mathematical control theory and finance, 149-159, Springer, Berlin 2008 . is linear in $(u, z, s)$ and independent of $x$. Hence,

$$
\begin{aligned}
& \tilde{x}(t)=t^{2} \tilde{x}(1), \tilde{x}(1) \in \mathbb{R}, \\
& \tilde{u}(t)=0
\end{aligned}
$$

gives, by Theorem 3.14 , the global minimum to the problem.

Example 3.16. Consider again the problem from Example 3.8. Replacing $x^{\nabla}$ by $u^{\rho}$ we can rewrite problem (10) as

$$
\begin{array}{r}
\operatorname{minimize} \mathcal{L}[x, u]=\int_{0}^{1}\left(\left(u^{\rho}(t)\right)^{2}+\alpha x^{2}(0)\right. \\
\left.+\beta(x(1)-1)^{2}\right) \nabla t
\end{array}
$$

subject to $x^{\nabla}(t)=u^{\rho}(t)$. Function $f$ is independent of $x$ and convex in $(u, z, s)$. The right-hand side of the control system is linear in $u$ and independent of $(x, z, s)$. Therefore, $\tilde{x}(t)=c(\alpha, \beta) t+\tilde{x}(0, \alpha, \beta)$ is, by Theorem 3.14, a global minimizer of the problem.

\section{Acknowledgements}

The authors were partially supported by the Center for Research and Development in Mathematics and Applications (CIDMA) of University of Aveiro via FCT and the EC fund FEDER/POCI 2010. ABM was also supported by Białystok University of Technology Grant S/WI/00/2011; DFMT by the Portugal-Austin project UTAustin/MAT/0057/2008.

[9] E. Girejko, A. B. Malinowska, and D. F. M. Torres, A unified approach to the calculus of variations on time scales, Proceedings of the 22nd Chinese Control and Decison Conference (2010 CCDC), Xuzhou, China, May 26-28, 2010. In: IEEE Catalog Number CFP1051D-CDR, 595-600 (2010).

[10] A. B. Malinowska, N. Martins, and D. F. M. Torres, Optim. Lett. 5, 41-53 (2011).

[11] A. B. Malinowska and D. F. M. Torres, Appl. Math. Comput. 217, 1158-1162 (2010).

[12] N. Martins and D. F. M. Torres, Discuss. Math. Differ. Incl. Control Optim. 31, in press (2011).

[13] R. Almeida and D. F. M. Torres, J. Vib. Control 15, 951-958 (2009).

[14] F. M. Atici, D. C. Biles, and A. Lebedinsky, Math. Comput. Modelling 43, 718-726 (2006).

[15] F. M. Atici and F. Uysal, Appl. Math. Lett. 21, 236-243 (2008). 
[16] P. A. F. Cruz, D. F. M. Torres, and A. S. I. Zinober, Int. J. Math. Modell. Numer. Optim. 1, 227-236 (2010).

[17] A. B. Malinowska and D. F. M. Torres, Math. Meth. Appl. Sci. 33, 1712-1722 (2010).

[18] N. Martins and D. F. M. Torres, Nonlinear Anal., Theory Methods Appl. Series A 71, e763-e773 (2009).

[19] E. Pawłuszewicz and D. F. M. Torres, Int. J. Control 83, 1573-1580 (2010).
[20] B. J. Jackson, Neural Parallel Sci. Comput. 16, 253272 (2008)

[21] M. Bohner and A. Peterson, Dynamic equations on time scales, Birkhäuser Boston, Boston, MA 2001.

[22] M. Bohner and A. Peterson, Advances in dynamic equations on time scales, Birkhäuser Boston, Boston, MA 2003. 